\title{
GEVREY-WELL-POSEDNESS FOR WEAKLY HYPERBOLIC OPERATORS WITH HÖLDER-CONTINUOUS COEFFICIENTS
}

\author{
F. COLOMBINI, D. DEL SANTO and T. KINOSHITA
}

\section{Introduction}

We shall consider the equation

$$
\partial_{t}^{2} u-\sum_{i, j=1}^{n} a_{i j}(t) \partial_{x_{i}} \partial_{x_{j}} u=0 \quad \text { in } \quad[0, T] \times \mathrm{R}_{x}^{n}
$$

with initial data

$$
u(0, x)=u_{0}(x), \quad \partial_{t} u(0, x)=u_{1}(x) \quad \text { in } \quad \mathrm{R}_{x}^{n},
$$

where $\left(a_{i j}(t)\right)_{1 \leq i, j \leq n}$ is a real symmetric matrix for all $t \in[0, T]$. Defining $a(t, \xi)=\sum_{i, j=1}^{n} a_{i j}(t) \xi_{i} \xi_{j} /|\xi|^{2}$, we will say that the equation (1.1) is strictly hyperbolic if $a(t, \xi)$ satisfies

$$
a(t, \xi) \geq{ }^{\exists} c_{0}>0 \quad \text { for all } t \in[0, T], \quad \xi \in \mathrm{R}_{\xi}^{n} \backslash\{0\} .
$$

We will say that the equation (1.1) is only weakly hyperbolic if

$$
a(t, \xi) \geq 0 \quad \text { for all } t \in[0, T], \quad \xi \in \mathrm{R}_{\xi}^{n} \backslash\{0\} .
$$

Throughout this paper the coefficients $a_{i j}(t)$ are supposed to be non-smooth: we assume that

$$
a_{i j}(t) \in C^{\alpha}([0, T]) \quad \text { for } \quad i, j=1,2, \ldots, n,
$$

where $C^{\alpha}([0, T])$ is the space of Hölder-continuous functions of order $\alpha$ on $[0, T]\left(f(t) \in C^{\alpha}([0, T])\right.$ if $f(t)$ has continuous derivatives on $[0, T]$ up to 
the order $[\alpha]$, the largest integer less or equal than $\alpha$, and

$$
\left.\|f\|_{C^{\alpha}([0, T])}=\sum_{k=0}^{[\alpha]} \sup _{t \in[0, T]}\left|f^{(k)}(t)\right|+\sup _{0 \leq s<t \leq T} \frac{\left|f^{([\alpha])}(t)-f^{([\alpha])}(s)\right|}{|t-s|^{\alpha-[\alpha]}}<+\infty\right) .
$$

Under the assumptions (1.3) and (1.5), if $\alpha \geq 1$, the Cauchy problem (1.1), (1.2) is well-posed in $C^{\infty}\left(\mathrm{R}_{x}^{n}\right)$, while, if $0<\alpha<1$, the problem (1.1), (1.2) is well-posed in $G^{s}\left(\mathrm{R}_{x}^{n}\right)$, the Gevrey class of order $s$, with $1 \leq s<\frac{1}{1-\alpha}$ (see [1]). Under the assumptions (1.4) and (1.5), if $0<\alpha<\infty$, the problem (1.1), (1.2) is well-posed in the Gevrey classes of order $1 \leq s<1+\frac{\alpha}{2}$ (see [6]). Some counter examples constructed using fast oscillatory coefficients show that these results cannot be improved from the point of view of the value of the Gevrey indexes.

We emphasize the fact that the regularity of the coefficients does not necessarily determine the speed of their oscillations. It is then natural to introduce, apart from the regularity, a new condition on the coefficients in order to control their oscillations. In our previous papers [2], [3] and [4] we assumed the oscillation condition as follows:

$$
\begin{cases}a_{i j}(t) \in C^{1}((0, T]) & \text { for } i, j=1,2, \ldots, n \\ \left|\partial_{t} a(t, \xi)\right| \leq \frac{{ }^{\exists} M}{t^{q}} & \text { for all } t \in(0, T], \xi \in \mathrm{R}_{\xi}^{n} \backslash\{0\},\end{cases}
$$

where $q>1-\alpha$. Assuming (1.3) (resp. (1.4)), (1.5) and (1.6), we showed that the Cauchy problem (1.1), (1.2) is well-posed in the Gevrey classes of order

$$
\begin{aligned}
& 1 \leq s<\left(\frac{q}{q-1}\right)\left(\frac{1}{1-\alpha}\right) \quad \text { if } \quad q>1 . \\
& \text { resp. }\left\{\begin{array}{ll}
1 \leq s \leq \frac{3}{2} & \text { if } q<3 \\
1 \leq s<\left(1+\frac{\alpha}{2}\right)\left(\frac{q}{q+\alpha-1}\right) & \text { if } q \geq 3
\end{array}\right)
\end{aligned}
$$

REMARK 1.1. As the parameter $q$ tends to infinity, the oscillation condition (1.6) loses sense and (1.7) and (1.8) approach to the results of [1] and [6].

REMARK 1.2. The oscillation speed is expressed in term of the first derivatives of the coefficients; therefore, as the exponent $\alpha$ of the regularity condition (1.5) tends to one, the oscillation condition (1.6) gives less effect to the wellposedness, and again (1.7) and (1.8) approach to the results of [1] and [6].

In the present paper, in order to control the speed of oscillation of the 
coefficients, we assume, instead of (1.6), the following condition: if $\beta \in \mathbf{N} \backslash\{0\}$,

$$
\begin{cases}a_{i j}(t) \in C^{\beta}(0, T] & \text { for } i, j=1,2, \ldots, n \\ \left|\partial_{t}^{\beta} a(t, \xi)\right| \leq \frac{{ }^{\exists} M}{t^{q}} & \text { for all } t \in(0, T], \xi \in \mathrm{R}_{\xi}^{n} \backslash\{0\},\end{cases}
$$

and, if $\beta \in(0,+\infty) \backslash \mathrm{N}$,

$$
\begin{cases}a_{i j}(t) \in C^{[\beta]}((0, T]) & \text { for } i, j=1,2, \ldots, n \\ \frac{\left|\partial_{t}^{[\beta]} a(s, \xi)-\partial_{t}^{[\beta]} a(t, \xi)\right|}{(s-t)^{\beta-[\beta]}} \leq \frac{{ }^{\exists} M}{t^{q}} & \text { for all } 0<t<s \leq T, \xi \in \mathrm{R}_{\xi}^{n} \backslash\{0\},\end{cases}
$$

REMARK 1.3. The condition (1.10) can be equivalently expressed in the following way: for all $\varepsilon \in(0, T), a_{i j}(t) \in C^{\beta}([\varepsilon, T])$ and there exists $M>0$ such that $\|a(\cdot, \xi)\|_{\left.C^{\beta}(\varepsilon, T]\right)} \leq M \varepsilon^{-q}$ for all $\varepsilon \in(0, T)$ and $\xi \in \mathrm{R}_{\xi}^{n} \backslash\{0\}$.

Assuming (1.5), without loss of generality, we can bound ourselves to consider in (1.9) and (1.10) only in the case when $q>\beta-\alpha$. Our first result concerns the strictly hyperbolic case.

Theorem 1.4. Let $T>0,0 \leq \alpha<1, \alpha<\beta$ and $q>\beta-\alpha$. Assume that (1.3), (1.5) and (1.9) or (1.10) hold. Then the Cauchy problem (1.1) and (1.2) is well-posed in the Gevrey classes of order

$$
1 \leq s< \begin{cases}(A)=\frac{q}{q(1-\alpha)+\alpha-\beta} & \text { if } q>\max \left\{1, \frac{\beta-\alpha}{1-\alpha}\right\} \\ (B)=\frac{1}{1-\beta} & \text { if } q \leq 1 \text { and } 0 \leq \alpha<\beta<1 .\end{cases}
$$

If $\beta \geq 1$ and $q<\frac{\beta-\alpha}{1-\alpha}$, the Cauchy problem (1.1) and (1.2) is well-posed in $C^{\infty}$

REMARK 1.5. When $\beta=1$, (1.11) coincides with (1.7).

Remark 1.6. As $q$ tends to infinity or $\beta$ tends to $\alpha,(1.11)$ approaches to the result of [1].

Let us turn now to the weakly hyperbolic case.

Theorem 1.7. Let $T>0$ and $q>\beta-\alpha$. Assume that (1.4), (1.5) and (1.9) or (1.10) hold. Then, if $0 \leq \alpha<\beta \leq 2$, the Cauchy problem (1.1), (1.2) 
is well-posed in the Gevrey classes of order

$$
1 \leq s< \begin{cases}(A)=\left(1+\frac{\alpha}{2}\right)\left(\frac{q}{q+\alpha-\beta}\right) & \text { if } q \geq \max \{3, \beta+2\}, \\ (B)=\frac{q\left(1+\frac{\alpha}{2}\right)+(q-3)(\alpha-\beta)}{q+(q-2)(\alpha-\beta)} & \text { if } \beta+2<q<3, \\ (C)=1+\frac{\beta}{2} & \text { otherwise. }\end{cases}
$$

Moreover, if $0 \leq \alpha<2<\beta$, the Cauchy problem (1.1), (1.2) is well-posed in the Gevrey classes of order

$$
1 \leq s< \begin{cases}(A)=\left(1+\frac{\alpha}{2}\right)\left(\frac{q}{q+\alpha-\beta}\right) & \text { if } q \geq \frac{4(\beta-\alpha)}{2-\alpha}, \\ (D)=\max \left\{2,\left(1+\frac{\alpha}{2}\right)\left(\frac{q+\beta+2}{q+\alpha+2}\right)\right\} & \text { if } q<\frac{4(\beta-\alpha)}{2-\alpha} .\end{cases}
$$

REMARK 1.8. When $\beta=1$, (1.12) coincides with (1.8).

REMARK 1.9. As $q$ tends to infinity or $\beta$ tends to $\alpha$, (1.12) and (1.13) approach to the result of [6].

REMARK 1.10. By the conditions (1.9) or (1.10) the oscillation of the coefficients is allowed, with the prescribed speed, near the point $t=0$. Actually it is possible to prove analogous well-posedness results when the oscillation occurs around any point $\bar{t} \in[0, T]$ and conditions similar to (1.9) or (1.10) are valid (see [2]).

The results of Theorem 1.4 and part of those of Theorem 1.7 are sharp. To see this we shall consider the Cauchy problem

$$
\begin{cases}\partial_{t}^{2} u-a(t) \partial_{x}^{2} u=0 & \text { in }[0,1] \times \mathrm{R}_{x}^{1} \\ u(0, x)=u_{0}(x), \quad \partial_{t} u(0, x)=u_{1}(x) & \text { in } \mathrm{R}_{x}^{1}\end{cases}
$$

and the oscillation of $a(t)$ will take place near $t=1$. The sharpness of the value $(B)$ of Theorem 1.4 is proved by the counter example constructed in [1]; the next result concernes only the value $(A)$.

TheOREM 1.11. Let $0 \leq \alpha<1, \alpha<\beta$ and $q>\beta-\alpha$. Then there exists $a$ function $a(t):[0,1] \rightarrow\left[\frac{1}{2}, \frac{3}{2}\right]$ belonging to $C^{\alpha}([0,1]) \cap C^{\beta}([0,1-\varepsilon])$ for 
all $\varepsilon \in(0,1)$ and satisfying

$$
\frac{\left|a^{([\beta])}(t)-a^{([\beta])}(s)\right|}{(t-s)^{\beta-[\beta]}} \leq \frac{{ }^{\exists} M}{(1-t)^{q}} \quad \text { for all } \quad 0 \leq s<t<1,
$$

and there exist $u_{0}(x), u_{1}(x) \in G^{s}\left(\mathrm{R}_{x}^{1}\right)$ such that the Cauchy problem (1.14) has no solution in $C^{1}\left([0,1] ;\left(D^{s}\right)^{\prime}\right)$ if

$$
s>\frac{q}{q(1-\alpha)+\alpha-\beta},
$$

where $\left(D^{s}\right)^{\prime}$ is the space of Gevrey ultradistributions of order s. In particular when $\beta \in \mathrm{N}$, (1.15) can be replaced by

$$
\left|a^{(\beta)}(t)\right| \leq \frac{{ }^{\exists} M}{(1-t)^{q}} \quad \text { for all } t \in[0,1) .
$$

Our last result shows that the value $(A)$ in Theorem 1.7 cannot be improved. We remark that the counter example of [6] proves that also the value $(C)$ of Theorem 1.7 is sharp.

THeOREM 1.12. Let $0 \leq \alpha<\beta$ and $q>\beta-\alpha$. Then there exists a function $a(t):[0,1] \rightarrow[0,2]$ belonging to $C^{\alpha}([0,1]) \cap C^{\beta}([0,1-\varepsilon])$ for all $\varepsilon \in(0,1)$ and satisfying the condition (1.15), and there exist $u_{0}(x), u_{1}(x) \in G^{s}\left(\mathbf{R}_{x}^{1}\right)$ such that the Cauchy problem (1.14) has no solution in $C^{1}\left([0,1] ;\left(D^{s}\right)^{\prime}\right)$ if

$$
s>\left(1+\frac{\alpha}{2}\right)\left(\frac{q}{q+\alpha-\beta}\right) .
$$

In particular when $\beta \in \mathrm{N}$, (1.15) can be replaced by (1.16).

The paper is organized as follows: in Paragraph 2 we state and prove some preliminary results on Hölder-continuous functions which will be used in the proofs on the main theorems. Paragraph 3 is devoted to the proof of the Theorem 1.4 and Paragraph 4 to that one of the Theorem 1.7. In the last paragraph we give a sketch of the proof of the Theorem 1.11 and only some ideas about that one of the Theorem 1.12, pointing out to the interested reader the similar constructions of counter examples in [1], [6], [7], [2], [3], [4] and [5].

\section{Preliminaries}

In this section we recall and we prove some lemmata of real analysis which characterize the functions satisfying the assumptions (1.9) or (1.10). 
Lemma 2.1 (Lemma 1 in [6]). Let $T>0$ and $\beta \geq 1$. Then there exists $M>0$ such that for all non-negative function $g(t)$ defined in $[0, T]$ and belonging to $C^{\beta}([\varepsilon, T])$ for all $\varepsilon \in(0, T)$, we have

$$
\int_{a}^{T}\left|\left\{g(t)^{1 / \beta}\right\}^{\prime}\right| d t \leq M\|g\|_{C^{\beta}[a, T]}^{1 / \beta} \quad \text { for all } \quad a \in(0, T) .
$$

From Lemma 2.1, we immediately get the following result.

Lemma 2.2. Let $T>0, \beta \geq 1$ and $q \geq 0$. Let $g(t)$ be a non-negative function defined in $[0, T]$ and belonging to $C^{\beta}([\varepsilon, T])$ for all $\varepsilon \in(0, T)$. Assume that there exists $M>0$ such that

$$
\begin{cases}\frac{\left|g^{([\beta])}(s)-g^{([\beta])}(t)\right|}{(s-t)^{\beta-[\beta]}} \leq \frac{M}{t^{q}} & \text { for all } 0<t<s \leq T, \text { if } \beta \text { is not an integer, } \\ \left|g(t)^{(\beta)}\right| \leq \frac{M}{t^{q}} & \text { for all } t \in(0, T], \text { if } \beta \text { is an integer. }\end{cases}
$$

Then there exists $M^{\prime}>0$ such that

$$
\int_{a}^{T}\left|\left\{g(t)^{1 / \beta}\right\}^{\prime}\right| d t \leq M^{\prime} a^{-q / \beta} \quad \text { for all } \quad a \in(0, T) .
$$

In the proofs of the Theorem 1.7 we will use, together with (2.2), another inequality. To obtain it we start quoting the following lemma.

Lemma 2.3 (Lemma 1 in [8]). Let $1<\beta \leq 2$. Then there exists $M>0$ such that for all non-negative function $g(t) \in C^{\beta}(\mathrm{R})$ we have

$$
\left|g(\sigma)^{\prime}\right| \leq M|g(\sigma)|^{1-1 / \beta}\left(\sup _{t<s} \frac{\left|g^{\prime}(s)-g^{\prime}(t)\right|}{(s-t)^{\beta-1}}\right)^{1 / \beta} \quad \text { for all } \quad \sigma \in \mathbf{R} .
$$

Lemma 2.3 is the key ingredient in the proof of the following result.

Lemma 2.4. Let $1<\beta \leq 2$. Then there exists $M^{\prime}>0$ such that for all $a, b \in \mathrm{R}$ with $a<b$, for all $\delta \in\left(0, \frac{b-a}{2}\right)$ and for all non-negative function $g(t) \in C^{\beta}([a, b])$ we have

$$
\left|g(t)^{\prime}\right| \leq M^{\prime}|g(t)|^{1-1 / \beta}\left(\|g\|_{C^{\beta}([a, b])}^{1 / \beta}+\delta^{1-\beta}\|g\|_{C^{1}([a, b])}\right)^{1 / \beta}
$$

for all $t \in[a+\delta, b-\delta]$. 
Proof. Let $\varphi(t)$ be a non-negative function belonging to $C^{\beta}(\mathrm{R})$ such that $\varphi(t)=1$ for all $t \geq 1$ and $\varphi(t)=0$ for all $t \leq 0$. Let us define

$$
\begin{aligned}
\tilde{g}(t)=\{1 & \left.-\varphi\left(\frac{t-a}{\delta}\right)\right\} g(a) \\
& +\left\{\varphi\left(\frac{t-a}{\delta}\right)+\varphi\left(\frac{b-t}{\delta}\right)-1\right\} g(t)+\left\{1-\varphi\left(\frac{b-t}{\delta}\right)\right\} g(b) .
\end{aligned}
$$

Since $\tilde{g}(t)$ is a non-negative function belonging to $C^{\beta}(\mathrm{R})$, by Lemma 2.3 we deduce that there exists $M>0$ such that, for all $\sigma \in \mathrm{R}$ satisfying $\tilde{g}(\sigma) \neq 0$,

$$
\left|\left\{\tilde{g}(\sigma)^{1 / \beta}\right\}^{\prime}\right| \leq M\left(\sup _{t<s} \frac{\left|\tilde{g}^{\prime}(s)-\tilde{g}^{\prime}(t)\right|}{(s-t)^{\beta-1}}\right)^{1 / \beta} .
$$

Noticing that

$$
\begin{aligned}
\tilde{g}^{\prime}(t) & =\frac{1}{\delta} \varphi^{\prime}\left(\frac{t-a}{\delta}\right)\{g(t)-g(a)\} \\
& +\frac{1}{\delta} \varphi^{\prime}\left(\frac{b-t}{\delta}\right)\{g(b)-g(t)\}+\left\{\varphi\left(\frac{t-a}{\delta}\right)+\varphi\left(\frac{b-t}{\delta}\right)-1\right\} g^{\prime}(t),
\end{aligned}
$$

we have

$$
\begin{aligned}
& \frac{\left|\tilde{g}^{\prime}(s)-\tilde{g}^{\prime}(t)\right|}{(s-t)^{\beta-1}} \\
& \leq \frac{\left|\frac{1}{\delta} \varphi^{\prime}\left(\frac{s-a}{\delta}\right)\{g(s)-g(a)\}-\frac{1}{\delta} \varphi^{\prime}\left(\frac{t-a}{\delta}\right)\{g(t)-g(a)\}\right|}{(s-t)^{\beta-1}} \\
& \quad+\frac{\left|\frac{1}{\delta} \varphi^{\prime}\left(\frac{b-s}{\delta}\right)\{g(b)-g(s)\}-\frac{1}{\delta} \varphi^{\prime}\left(\frac{b-t}{\delta}\right)\{g(b)-g(t)\}\right|}{(s-t)^{\beta-1}} \\
& \quad+\frac{\left|\left\{\varphi\left(\frac{s-a}{\delta}\right)+\varphi\left(\frac{b-s}{\delta}\right)-1\right\} g^{\prime}(s)-\left\{\varphi\left(\frac{t-a}{\delta}\right)+\varphi\left(\frac{b-t}{\delta}\right)-1\right\} g^{\prime}(t)\right|}{(s-t)^{\beta-1}} \\
& \equiv I+I I+I I I .
\end{aligned}
$$

We first estimate $I$. We remark that the support of $\varphi^{\prime}\left(\frac{t-a}{\delta}\right)$ is contained in $[a, a+\delta]$. We shall consider two different cases. Suppose first that $a \leq t<$ $a+\delta<s$. In this case we have that $\varphi^{\prime}\left(\frac{s-a}{\delta}\right)=0$. Since $s>a+\delta$ and 
$\delta>t-a$, using also the fact that $\varphi^{\prime}(1)=0$, we obtain

$$
\begin{aligned}
I & =\frac{\left|\frac{1}{\delta} \varphi^{\prime}\left(\frac{t-a}{\delta}\right)\{g(t)-g(a)\}\right|}{(s-t)^{\beta-1}}=\frac{\left|\varphi^{\prime}\left(\frac{t-a}{\delta}\right)\right|}{(s-t)^{\beta-1}} \cdot \frac{|g(t)-g(a)|}{\delta} \\
& \leq \frac{\left|\varphi^{\prime}\left(\frac{t-a}{\delta}\right)\right|}{(a+\delta-t)^{\beta-1}} \cdot \frac{|g(t)-g(a)|}{t-a} \\
& \leq \frac{1}{\delta^{\beta-1}} \cdot \frac{\left|\varphi^{\prime}(1)-\varphi^{\prime}\left(\frac{t-a}{\delta}\right)\right|}{\left(1-\frac{t-a}{\delta}\right)^{\beta-1}} \cdot \frac{|g(t)-g(a)|}{t-a} \\
& \leq \delta^{1-\beta}\|\varphi\|_{C^{\beta}(\mathrm{R})}\|g\|_{C^{1}([a, b])} .
\end{aligned}
$$

Next suppose $a \leq t<s<a+\delta$. Noticing that $s-t \leq \delta$ and $s-a \leq \delta$, we deduce

$$
\begin{aligned}
I \leq & \frac{1}{\delta} \cdot\left|\varphi^{\prime}\left(\frac{t-a}{\delta}\right)\right| \cdot \frac{|g(s)-g(t)|}{(s-t)^{\beta-1}}+\frac{1}{\delta} \cdot \frac{\left|\varphi^{\prime}\left(\frac{s-a}{\delta}\right)-\varphi^{\prime}\left(\frac{t-a}{\delta}\right)\right|}{(s-t)^{\beta-1}} \cdot|g(s)-g(a)| \\
\leq & \frac{1}{\delta} \cdot\left|\varphi^{\prime}\left(\frac{t-a}{\delta}\right)\right| \cdot \frac{(s-t)\left|g^{\prime}\left(s+{ }^{\exists} \theta(t-s)\right)\right|}{(s-t)^{\beta-1}} \\
& \quad+\frac{s-a}{\delta^{\beta}} \cdot \frac{\left|\varphi^{\prime}\left(\frac{s-a}{\delta}\right)-\varphi^{\prime}\left(\frac{t-a}{\delta}\right)\right|}{\left(\frac{s-a}{\delta}-\frac{t-a}{\delta}\right)^{\beta-1}} \cdot \frac{|g(s)-g(a)|}{s-a} \\
\leq & \frac{1}{\delta}(s-t)^{2-\beta}\|\varphi\|_{C^{1}(\mathrm{R})}\|g\|_{C^{1}([a, b])}+\frac{s-a}{\delta^{\beta}}\|\varphi\|_{C^{\beta}(\mathrm{R})}\|g\|_{C^{1}([a, b])} \\
\leq & 2 \delta^{1-\beta}\|\varphi\|_{C^{\beta}(\mathrm{R})}\|g\|_{C^{1}([a, b])} .
\end{aligned}
$$

Therefore we get

$$
I \leq 2 \delta^{1-\beta}\|\varphi\|_{C^{\beta}(\mathrm{R})}\|g\|_{C^{1}[a, b]} .
$$

Similarly we get

$$
I I \leq 2 \delta^{1-\beta}\|\varphi\|_{C^{\beta}(\mathrm{R})}\|g\|_{C^{1}[a, b]} .
$$

To estimate $I I I$ we remark that the supports of $\left\{\varphi\left(\frac{s-a}{\delta}\right)+\varphi\left(\frac{b-s}{\delta}\right)-1\right\}$ and $\left\{\varphi\left(\frac{t-a}{\delta}\right)+\varphi\left(\frac{b-t}{\delta}\right)-1\right\}$ are contained in $[a, b]$ and consequently we may only consider the case of $a \leq t<s \leq b$. We deduce 


$$
\begin{aligned}
I I I \leq & \frac{\left|\varphi\left(\frac{s-a}{\delta}\right)-\varphi\left(\frac{t-a}{\delta}\right)\right|\left|g^{\prime}(t)\right|}{(s-t)^{\beta-1}}+\frac{\left|\varphi\left(\frac{b-s}{\delta}\right)-\varphi\left(\frac{b-t}{\delta}\right)\right|\left|g^{\prime}(t)\right|}{(s-t)^{\beta-1}} \\
& +\frac{\left|\varphi\left(\frac{s-a}{\delta}\right)+\varphi\left(\frac{b-s}{\delta}\right)-1\right|\left|g^{\prime}(s)-g^{\prime}(t)\right|}{(s-t)^{\beta-1}} \\
\leq & \frac{\left|\varphi\left(\frac{s-a}{\delta}\right)-\varphi\left(\frac{t-a}{\delta}\right)\right|}{\left(\frac{s-a}{\delta}-\frac{t-a}{\delta}\right)^{\beta-1}} \cdot \frac{\left|g^{\prime}(t)\right|}{\delta^{\beta-1}}+\frac{\left|\varphi\left(\frac{b-s}{\delta}\right)-\varphi\left(\frac{b-t}{\delta}\right)\right|}{\left(\frac{s-a}{\delta}-\frac{t-a}{\delta}\right)^{\beta-1}} \cdot \frac{\left|g^{\prime}(t)\right|}{\delta^{\beta-1}} \\
& \quad+\frac{\left|g^{\prime}(s)-g^{\prime}(t)\right|}{(s-t)^{\beta-1}} \\
\leq & 2 \delta^{1-\beta}\|\varphi\|_{C^{\beta-1}(\mathrm{R})}\|g\|_{C^{1}([a, b])}+\|g\|_{C^{\beta}([a, b])}
\end{aligned}
$$

Thus, by (2.6)-(2.9) it follows that

$$
\sup _{t<s} \frac{\left|\tilde{g}^{\prime}(s)-\tilde{g}^{\prime}(t)\right|}{(s-t)^{\beta-1}} \leq 6 \delta^{1-\beta}\|\varphi\|_{C^{\beta}(\mathrm{R})}\|g\|_{C^{1}([a, b])}+\|g\|_{C^{\beta}([a, b])} .
$$

This together with (2.5) implies (2.4), since $\left|\left\{g(t)^{1 / \beta}\right\}^{\prime}\right|=\left|\left\{\tilde{g}(t)^{1 / \beta}\right\}^{\prime}\right|$ for all $t \in[a+\delta, b-\delta]$ satisfying $g(t) \neq 0$.

Lemma 2.5. Let $T>0,0<\gamma \leq 1$ and $\gamma<q$. Let $h(t)$ be a function defined in $[0, T]$ and belonging to $C^{\gamma}([\varepsilon, T])$ for all $\varepsilon \in(0, T)$. Assume that there exists $M>0$ such that

$$
\begin{cases}\frac{|h(s)-h(t)|}{(s-t)^{\gamma}} \leq \frac{M}{t^{q}} & \text { for all } 0<t<s \leq T \quad \text { if } 0<\gamma<1, \\ \left|h^{\prime}(t)\right| \leq \frac{M}{t^{q}} & \text { for all } t \in(0, T] \quad \text { if } \gamma=1 .\end{cases}
$$

Then there exists $\tilde{M}>0$ such that

$$
|h(t)| \leq \frac{\tilde{M}}{t^{q-\gamma}} \quad \text { for all } \quad t \in(0, T]
$$

Proof. Since the case of $\gamma=1$ is trivial, we suppose that $0<\gamma<1$. Let $0<t_{0} \leq T / 2$. We remark that there exists $n_{0} \in \mathrm{N} \backslash\{0\}$ such that $T / 2^{n_{0}+1} \leq t_{0} \leq T / 2^{n_{0}}$. Since $\left|h\left(t_{0}\right)-h\left(2 t_{0}\right)\right| \leq M t_{0}^{-q+\gamma}$, we have

$$
\left|h\left(t_{0}\right)\right| \leq\left|h\left(2 t_{0}\right)\right|+M t_{0}^{-q+\gamma},
$$

and similarly

$$
\left|h\left(2 t_{0}\right)\right| \leq\left|h\left(4 t_{0}\right)\right|+M\left(2 t_{0}\right)^{-q+\gamma} .
$$


Recursively, we deduce that

$$
\left|h\left(t_{0}\right)\right| \leq\left|h\left(2^{n_{0}} t_{0}\right)\right|+M t_{0}^{-q+\gamma} \sum_{j=0}^{n_{0}-1} \frac{1}{\left(2^{q-\gamma}\right)^{j}} .
$$

Hence we get for all $t \in(0, T / 2]$

$$
|h(t)| \leq \sup _{T / 2 \leq s \leq T}|h(s)|+\frac{M}{1-2^{-q+\gamma}} t^{-q+\gamma} .
$$

From this the inequality (2.10) follows easily.

Lemma 2.6. Let $T>0,1<\beta \leq 2$ and $q \geq \beta-1$. Let $g(t)$ be $a$ non-negative function defined in $[0, T]$ and belonging to $C^{\beta}([\varepsilon, T])$ for all $\varepsilon \in(0, T)$. Assume that there exists $M>0$ such that

$$
\begin{cases}\frac{\left|g^{\prime}(s)-g^{\prime}(t)\right|}{(s-t)^{\beta-1}} \leq \frac{M}{t^{q}} & \text { for all } 0<t<s \leq T \quad \text { if } 1<\beta<2, \\ \left|g^{\prime \prime}(t)\right| \leq \frac{M}{t^{q}} & \text { for all } t \in(0, T] \quad \text { if } \beta=2 .\end{cases}
$$

Then there exists $M^{\prime}>0$ such that

$$
\left|g^{\prime}(t)\right| \leq M^{\prime}|g(t)|^{1-1 / \beta} t^{-q / \beta} \quad \text { for all } t \in(0, T / 2) .
$$

PRoOF. We take an arbitrarily fixed point $t_{0} \in(0, T / 2)$. Setting $a=t_{0} / 2$, $b=T-t_{0} / 2$ and $\delta=t_{0} / 2$, by Lemma 2.4 we find that there exists $M^{\prime}>0$ such that for $t \in\left[t_{0}, T-t_{0}\right]$ satisfying $g(t) \neq 0$

$$
\left|\left\{g(t)^{1 / \beta}\right\}^{\prime}\right| \leq M^{\prime}\left(\|g\|_{C^{\beta}\left(\left[t_{0} / 2, T-t_{0} / 2\right]\right)}+\left(\frac{t_{0}}{2}\right)^{1-\beta}\|g\|_{\left.C^{1}\left(t_{0} / 2, T-t_{0} / 2\right]\right)}\right)^{1 / \beta} .
$$

Applying Lemma 2.5 to the function $g^{\prime}(t)$ we deduce that

$$
\left|g^{\prime}(t)\right| \leq \frac{\tilde{M}}{t^{q-\beta+1}} \quad \text { for all } \quad t \in(0, T],
$$

and consequently

$$
\|g\|_{C^{1}\left(\left[t_{0} / 2, T-t_{0} / 2\right]\right)} \leq M_{1} t_{0}^{-q+\beta-1} .
$$

On the other hand (2.11) implies that

$$
\|g\|_{C^{\beta}\left(\left[t_{0} / 2, T-t_{0} / 2\right]\right)} \leq M_{2} t_{0}^{-q} .
$$


Consequently

$$
\left|\left\{g(t)^{1 / \beta}\right\}^{\prime}\right| \leq M t_{0}^{-q / \beta} \text { for all } t \in\left[t_{0}, T-t_{0}\right] \text { such that } g(t) \neq 0 .
$$

In particular

$$
\left|\left\{g\left(t_{0}\right)^{1 / \beta}\right\}^{\prime}\right| \leq M t_{0}^{-q / \beta}
$$

and (2.12) follows .

Lemma 2.7. Let $T>0$ and $\alpha, v, \beta \in(0,+\infty)$ with $\alpha<v<\beta$. Let $g(t)$ be a function defined in $[0, T]$ and belonging to $C^{\alpha}[0, T] \cap C^{\beta}[\varepsilon, T]$ for all $\varepsilon \in(0, T)$. Assume that there exists $M>0$ such that

$$
\begin{cases}\frac{\left|\partial_{t}^{[\beta]} g(s)-\partial_{t}^{[\beta]} g(t)\right|}{(s-t)^{\beta-[\beta]}} \leq \frac{M}{t^{q}} & \text { for all } 0<t<s \leq T \quad \text { if } \beta \text { is not an integer } \\ \left|\partial_{t}^{\beta} g(t)\right| \leq \frac{M}{t^{q}} & \text { for all } t \in(0, T] \quad \text { if } \beta \text { is an integer. }\end{cases}
$$

Then, if $v$ is not an integer, there exists $M^{\prime}>0$ such that

$$
\frac{\left|\partial_{t}^{[\nu]} g(s)-\partial_{t}^{[v]} g(t)\right|}{(s-t)^{\nu-[\nu]}} \leq \frac{M^{\prime}}{t^{\frac{q(\nu-\alpha)}{\beta-\alpha}}} \quad \text { for all } \quad 0<t<s \leq T .
$$

Proof. We fix a point $t_{0}$ in the interval $(0, T)$. Since $v$ is not an integer, by the generalized Kolmogorov inequality (see [9]) we find that

$$
\|g\|_{C^{v}\left(\left[t_{0}, T\right]\right)} \leq C\|g\|_{C^{\alpha}\left(\left[t_{0}, T\right]\right)}^{1-\frac{\nu-\alpha}{\beta-\alpha}}\|g\|_{C^{\beta}\left(\left[t_{0}, T\right]\right)}^{\frac{\nu-\alpha}{\beta-\alpha}} \leq C\left(\frac{M}{t_{0}^{q}}\right)^{\frac{\nu-\alpha}{\beta-\alpha}}=\frac{M^{\prime}}{t_{0}^{\frac{q(\nu-\alpha)}{\beta-\alpha}}}
$$

In particular, it also holds that

$$
\frac{\left|\partial_{t}^{[v]} g(s)-\partial_{t}^{[\nu]} g\left(t_{0}\right)\right|}{\left(s-t_{0}\right)^{\nu-[v]}} \leq \frac{M^{\prime}}{t_{0}^{\frac{q(\nu-\alpha)}{\beta-\alpha}}} \quad \text { for all } \quad t_{0}<s \leq b .
$$

Hence we get (2.13), since $t_{0}$ is an arbitrarily fixed point in $(0, T)$.

\section{Proof of Theorem 1.4}

When $s=1$, the Cauchy problem (1.1) and (1.2) is well-posed in $G^{1}$ which is the topological vector space of analytic functions on $\mathrm{R}_{x}^{n}$ (see $\$ 8$ in [1]). Therefore we can devote ourselves to the proof when the Gevrey order $s$ is larger than 1; Moreover we suppose that the initial data $u_{0}(x)$ and $u_{1}(x)$ belong to $G^{s}\left(\mathrm{R}_{x}^{n}\right)$ and have compact support. Our task is to investigate the regularity 
of the solution and this will be done by the usual way of energy estimates and Paley-Wiener theorem.

By Fourier transform with respect to the space variable the Cauchy problem (1.1) and (1.2) is changed into

$$
\partial_{t}^{2} v+a(t, \xi)|\xi|^{2} v=0 \quad \text { in } \quad[0, T] \times \mathrm{R}_{\xi}^{n}
$$

with initial data

$$
v(0, \xi)=v_{0}(\xi) \quad \partial_{t} v(0, \xi)=v_{1}(\xi) \quad \text { in } \quad \mathrm{R}_{\xi}^{n} .
$$

We define the energy as

$$
E(t, \xi)=\tilde{a}(t, \xi)|\xi|^{2}|v|^{2}+\left|\partial_{t} v\right|^{2},
$$

where $\tilde{a}(t, \xi)\left(\geq{ }^{\exists} c_{0}>0\right)$ is a differentable fuction which will be defined later. Differentiating $E(t, \xi)$ with respect to the time and using the equation (3.1), we have

$$
\begin{aligned}
\partial_{t} E(t, \xi) & =2 \tilde{a}(t, \xi)|\xi|^{2} \Re\left(\partial_{t} v, v\right)+\partial_{t} \tilde{a}(t, \xi)|\xi|^{2}|v|^{2}+2 \Re\left(\partial_{t}^{2} v, \partial_{t} v\right) \\
& =2\{\tilde{a}(t, \xi)-a(t, \xi)\}|\xi|^{2} \Re\left(\partial_{t} v, v\right)+\frac{\partial_{t} \tilde{a}(t, \xi)}{\tilde{a}(t, \xi)} \tilde{a}(t, \xi)|\xi|^{2}|v|^{2} \\
& \leq \frac{|\tilde{a}(t, \xi)-a(t, \xi)||\xi|}{\sqrt{\tilde{a}(t, \xi)}} E(t, \xi)+\frac{\left|\partial_{t} \tilde{a}(t, \xi)\right|}{\tilde{a}(t, \xi)} E(t, \xi) \text { for } t \in[0, T] .
\end{aligned}
$$

Hence Gronwall's inequality yields

$$
E(t, \xi) \leq E(0, \xi) \exp \left\{\int_{0}^{T} \frac{|\tilde{a}(t, \xi)-a(t, \xi)|}{\sqrt{\tilde{a}(t, \xi)}} d t|\xi|+\int_{0}^{T} \frac{\left|\partial_{t} \tilde{a}(t, \xi)\right|}{\tilde{a}(t, \xi)} d t\right\}
$$

for all $t \in[0, T]$. By (1.3) we get

$$
E(t, \xi) \leq E(0, \xi) \exp \left\{\frac{1}{\sqrt{c_{0}}} \int_{0}^{T}|\tilde{a}(t, \xi)-a(t, \xi)| d t|\xi|+\frac{1}{c_{0}} \int_{0}^{T}\left|\partial_{t} \tilde{a}(t, \xi)\right| d t\right\}
$$

for all $t \in[0, T]$. We extend the set of definition of the coefficients to the whole $\mathrm{R}$ in such a way that $a_{i, j}(t)=0$ for all $t \in(-\infty,-1] \cup[T+1, \infty)$ and $a_{i, j}(t) \in C^{\alpha}(\mathrm{R}) \cap C^{\beta}((0,+\infty))$. We separate the proof into two parts according to the case that $0 \leq \alpha<\beta \leq 1$, or $0 \leq \alpha<1<\beta$.

Let then $0 \leq \alpha<\beta \leq 1$. We need the following result.

Lemma 3.1. Let $0<\varepsilon \leq 1$ and $\rho(t)$ be a non-negative function such that $\rho(t) \in C_{0}^{\infty}(\mathrm{R})$, supp $\rho \subset[-1,1]$, and $\int_{-\infty}^{\infty} \rho(\tau) d \tau=1$. Let $a_{\varepsilon}(t, \xi)=$ 
$\int_{-\infty}^{\infty} a(t+\tau \varepsilon, \xi) \rho(\tau) d \tau$. If $a(t, \xi)$ satisfies (1.5) and (1.10) (or (1.9)) with $0 \leq \alpha<\beta \leq 1$, then there exist $L>0$ and $K>0$ such that

$$
\left|a_{\varepsilon}(t, \xi)-a(t, \xi)\right| \leq L \varepsilon^{\alpha}, \quad\left|\partial_{t} a_{\varepsilon}(t, \xi)\right| \leq K \varepsilon^{\alpha-1}
$$

for all $t \in[0, T]$,

$$
\left|a_{\varepsilon}(t, \xi)-a(t, \xi)\right| \leq L t^{-q} \varepsilon^{\beta}, \quad\left|\partial_{t} a_{\varepsilon}(t, \xi)\right| \leq K t^{-q} \varepsilon^{\beta-1}
$$

for all $t \in\left[\frac{3}{2} \varepsilon, T\right]$.

PRoof. Let us prove for instance (3.6). By (1.10) (or (1.9)) we get for $\frac{3}{2} \varepsilon \leq t \leq T$

$$
\begin{aligned}
&\left|a_{\varepsilon}(t, \xi)-a(t, \xi)\right|\left|\int_{-\infty}^{\infty} a(t+\tau \varepsilon, \xi) \rho(\tau) d \tau-\int_{-\infty}^{\infty} a(t, \xi) \rho(\tau) d \tau\right| \\
& \leq \int_{-1}^{1}|a(t+\tau \varepsilon, \xi)-a(t, \xi)| \rho(\tau) d \tau \\
& \leq \int_{-1}^{1} \frac{M}{(t-\varepsilon)^{q}}|\tau \varepsilon|^{\beta} \rho(\tau) d \tau \\
& \leq \frac{M}{\left(t-\frac{2}{3} t\right)^{q}} \varepsilon^{\beta} \int_{-1}^{1}|\tau|^{\beta} \rho(\tau) d \tau \leq L t^{-q} \varepsilon^{\beta} \\
&\left|\partial_{t} a_{\varepsilon}(t, \xi)\right|=\left|\frac{1}{\varepsilon} \int_{-\infty}^{\infty} a(t+\tau \varepsilon, \xi) \rho^{\prime}(\tau) d \tau-\frac{1}{\varepsilon} \int_{-\infty}^{\infty} a(t, \xi) \rho^{\prime}(\tau) d \tau\right| \\
& \leq \frac{1}{\varepsilon} \int_{-1}^{1}|a(t+\tau \varepsilon, \xi)-a(t, \xi)|\left|\rho^{\prime}(\tau)\right| d \tau \\
& \leq \frac{1}{\varepsilon} \int_{-1}^{1} \frac{M}{(t-\varepsilon)^{q}}|\tau \varepsilon|^{\beta}\left|\rho^{\prime}(\tau)\right| d \tau \\
& \leq \frac{M}{\left(t-\frac{2}{3} t\right)^{q}} \varepsilon^{\beta-1} \int_{-1}^{1}|\tau|^{\beta}\left|\rho^{\prime}(\tau)\right| d \tau \leq K t^{-q} \varepsilon^{\beta-1} .
\end{aligned}
$$

Let us come again to the proof of the Theorem 1.4. We define

$$
\tilde{a}(t, \xi)=a_{\varepsilon}(t, \xi)=\int_{-\infty}^{\infty} a(t+\tau \varepsilon, \xi) \rho(\tau) d \tau
$$


where $0<\varepsilon \leq 1$. If $\frac{3}{2} \varepsilon<\delta \leq T$, by (3.5) and (3.6) we obtain

$$
\begin{aligned}
& \int_{0}^{T}|\tilde{a}(t, \xi)-a(t, \xi)| d t \leq \int_{0}^{\delta}\left|a_{\varepsilon}(t, \xi)-a(t, \xi)\right| d t+\int_{\delta}^{T}\left|a_{\varepsilon}(t, \xi)-a(t, \xi)\right| d t \\
& \leq \int_{0}^{\delta} L \varepsilon^{\alpha} d t+\int_{\delta}^{T} L t^{-q} \varepsilon^{\beta} d t \\
& \leq \begin{cases}C\left\{\varepsilon^{\alpha} \delta+\varepsilon^{\beta} \log \delta^{-1}\right\} & \text { if } 0<q \leq 1 \\
C\left\{\varepsilon^{\alpha} \delta+\varepsilon^{\beta} \delta^{1-q}\right\} & \text { if } 1<q<\infty .\end{cases} \\
& \int_{0}^{T}\left|\partial_{t} \tilde{a}(t, \xi)\right| d t \leq \int_{0}^{\delta}\left|\partial_{t} a_{\varepsilon}(t, \xi)\right| d t+\int_{\delta}^{T}\left|\partial_{t} a_{\varepsilon}(t, \xi)\right| d t \\
& \leq \int_{0}^{\delta} K \varepsilon^{\alpha-1} d t+\int_{\delta}^{T} K t^{-q} \varepsilon^{\beta-1} d t \\
& \leq \begin{cases}C\left(\varepsilon^{\alpha-1} \delta+\varepsilon^{\beta-1} \log \delta^{-1}\right) & \text { if } 0<q \leq 1 \\
C\left(\varepsilon^{\alpha-1} \delta+\varepsilon^{\beta-1} \delta^{1-q}\right) & \text { if } 1<q<\infty .\end{cases}
\end{aligned}
$$

Taking $\delta=\left\{\begin{array}{ll}\varepsilon^{\beta-\alpha} & \text { if } 0<q \leq 1 \\ \varepsilon^{\frac{\beta-\alpha}{q}} & \text { if } 1<q<\infty,\end{array}\right.$ by (3.7) and (3.8) we get

$$
\begin{aligned}
\int_{0}^{T} \mid \tilde{a}(t, \xi) & -a(t, \xi) \mid d t \\
& \leq\left\{\begin{array}{ll}
C \varepsilon^{\beta} \log \varepsilon^{-1} & \text { if } 0<q \leq 1 \\
C \varepsilon^{\alpha+\frac{\beta-\alpha}{q}} & \text { if } 1<q<\infty
\end{array} \leq C \varepsilon^{\alpha+\frac{\beta-\alpha}{\max \{q, 1]} \log \varepsilon^{-1},}\right. \\
\int_{0}^{T}\left|\partial_{t} \tilde{a}(t, \xi)\right| d t & \leq\left\{\begin{array}{ll}
C \varepsilon^{\beta-1} \log \varepsilon^{-1} & \text { if } 0<q \leq 1 \\
C \varepsilon^{\alpha-1+\frac{\beta-\alpha}{q}} & \text { if } 1<q<\infty
\end{array} \leq C \varepsilon^{\alpha-1+\frac{\beta-\alpha}{\max (q, 1]} \log \varepsilon^{-1} .}\right.
\end{aligned}
$$

Therefore by (3.4) we deduce that

$$
E(t, \xi) \leq E(0, \xi) \exp \left\{C\left(|\xi|+\varepsilon^{-1}\right) \varepsilon^{\alpha+\frac{\beta-\alpha}{\max (q, 1)}} \log \varepsilon^{-1}\right\} \quad \text { for all } t \in[0, T]
$$


Finally we choose the parameter $\varepsilon$ such that $|\xi|=\varepsilon^{-1}$. Then we get the following energy inequality

$$
E(t, \xi) \leq E(0, \xi) \exp \left\{C|\xi|^{\left.1-\alpha-\frac{\beta-\alpha}{\max (q, 1)} \log |\xi|\right\}}\right.
$$

$$
\left.\leq E(0, \xi) \exp \left\{C|\xi|^{\max \left\{\frac{q(1-\alpha)+\alpha-\beta}{q}, 1-\beta\right.}\right\} \log |\xi|\right\} \quad \text { for all } t \in[0, T]
$$

We consider now the case that $0 \leq \alpha<1<\beta$. Taking $v \in(\alpha, 1)$, from (2.13) we deduce that

$$
\frac{|a(s, \xi)-a(t, \xi)|}{(s-t)^{v}} \leq \frac{{ }^{\exists} M^{\prime}}{t^{\frac{q(v-\alpha)}{\beta-\alpha}}} \quad \text { for all } \quad 0<t<s \leq T, \quad \xi \in \mathrm{R}_{\xi}^{n} \backslash\{0\} .
$$

Arguing as in the previous case, with $\beta$ and $q$ replaced by $v$ and $\frac{q(v-\alpha)}{\beta-\alpha}$ respectively, we obtain

$$
\begin{aligned}
E(t, \xi) & \leq E(0, \xi) \exp \left\{C|\xi|^{1-\alpha-\frac{v-\alpha}{\max (q(v-\alpha) /(\beta-\alpha), 1\}}} \log |\xi|\right\} \\
& =E(0, \xi) \exp \left\{C|\xi|^{\max \left\{\frac{q(1-\alpha)+\alpha-\beta}{q}, 1-v\right\}} \log |\xi|\right\} \text { for all } t \in[0, T]
\end{aligned}
$$

Hence, if $q>\frac{\beta-\alpha}{1-\alpha}$ we have, taking $v=\alpha+\frac{\beta-\alpha}{q}$,

$$
E(t, \xi) \leq E(0, \xi) \exp \left\{C|\xi|^{\frac{q(1-\alpha)+\alpha-\beta}{q}} \log |\xi|\right\} \quad \text { for } \quad t \in[0, T] .
$$

Thus, by (3.9) and (3.10) we get the energy inequality

$$
\begin{aligned}
& E(t, \xi) \\
& \leq \begin{cases}E(0, \xi) \exp \left\{C|\xi|^{\frac{q(1-\alpha)+\alpha-\beta}{q}} \log |\xi|\right\} & \text { if } q \geq \max \left\{1, \frac{\beta-\alpha}{1-\alpha}\right\} \\
E(0, \xi) \exp \left\{C|\xi|^{1-\beta} \log |\xi|\right\} & \text { if } 0 \leq \alpha<\beta<1, \beta-\alpha<q \leq 1 .\end{cases}
\end{aligned}
$$

In virtue of Paley-Wiener theorem, $\{u(\cdot, t) ; t \in[0, T]\}$ is bounded in the Gevrey classes of order (1.11).

If $\beta>1$ and $q<\frac{\beta-\alpha}{1-\alpha}$, there exists $1<v(<2)$ such that $q \leq \frac{\beta-\alpha}{\nu-\alpha}$. By (2.13) we find that

$$
\frac{\left|\partial_{t} a(s, \xi)-\partial_{t} a(t, \xi)\right|}{(s-t)} \leq \frac{M^{\prime}}{t} \quad \text { for all } \quad 0<t<s \leq T, \quad \xi \in \mathrm{R}_{\xi}^{n} \backslash\{0\},
$$

and then

$$
\left|\partial_{t}^{\beta} a(t, \xi)\right| \leq \frac{{ }^{\exists} M^{\prime \prime}}{t} \quad \text { for all } \quad 0<t \leq T, \quad \xi \in \mathrm{R}_{\xi}^{n} \backslash\{0\} .
$$


From [3] (see also [2]) we obtain the well-posedness in $C^{\infty}$. This concludes the proof of Theorem 1.4.

\section{Proof of Theorem 1.7}

In this section we will prove the Theorem 1.7. The strategy will be the same as in the previous proof: we will use the energy inequalities and the PaleyWiener Theorem. To obtain the energy inequalities we will divide the proof into several parts according to the following cases: Case $1.0 \leq \alpha<\beta \leq 1$, Case $2.0 \leq \alpha \leq 1<\beta \leq 2$, Case 3. $1<\alpha<\beta \leq 2$, Case $4.0<\alpha<2<\beta$.

Case 1. $0 \leq \alpha<\beta \leq 1$

Since the coefficients may be not differentiable, they cannot enter directly into the definition of the energy. As in the proof of Theorem 1.4 we regularize the coefficients using Friedrichs' mollifier: we put $a_{\varepsilon}(t, \xi)=\int_{-\infty}^{\infty} a(t+$ $\tau \varepsilon, \xi) \rho(\tau) d \tau$ and we define

$$
\tilde{a}(t, \xi)= \begin{cases}a_{\varepsilon}(t, \xi)+\varepsilon^{\alpha} & \text { for } 0 \leq t \leq \delta \\ a_{\varepsilon}(t, \xi)+t^{-\max \left\{2, \frac{2 q}{3}\right\}} \varepsilon^{\gamma} & \text { for } \delta \leq t \leq T,\end{cases}
$$

where $\frac{3}{2} \varepsilon \leq \delta \leq T$ and $\gamma \geq 0$. By (3.5) and (3.6) we obtain

$$
\begin{aligned}
\int_{0}^{T} \frac{|\tilde{a}(t, \xi)-a(t, \xi)|}{\sqrt{\tilde{a}(t, \xi)}} d t & \int_{0}^{\delta} \frac{\left|a_{\varepsilon}-a+\varepsilon^{\alpha}\right|}{\sqrt{\tilde{a}}} d t+\int_{\delta}^{T} \frac{\left|a_{\varepsilon}-a+t^{-\max \left\{2, \frac{2 q}{3}\right\}} \varepsilon^{\gamma}\right|}{\sqrt{\tilde{a}}} d t \\
\quad \leq & \int_{0}^{\delta} \frac{L \varepsilon^{\alpha}+\varepsilon^{\alpha}}{\sqrt{\varepsilon^{\alpha}}} d t+\int_{\delta}^{T} \frac{L t^{-q} \varepsilon^{\beta}+t^{-\max \left\{2, \frac{2 q}{3}\right\}} \varepsilon^{\gamma}}{\sqrt{t^{-\max \left\{2, \frac{2 q}{3}\right\}} \varepsilon^{\gamma}}} d t \\
& \leq(L+1) \varepsilon^{\frac{\alpha}{2}} \int_{0}^{\delta} d t+L \varepsilon^{\beta-\frac{\gamma}{2}} \int_{\delta}^{T} t^{-\min \left\{q-1, \frac{2 q}{3}\right\}} d t+\varepsilon^{\frac{\gamma}{2}} \int_{\delta}^{T} t^{-\max \left\{1, \frac{q}{3}\right\}} d t \\
(4.1) \leq & \begin{cases}C\left(\varepsilon^{\frac{\alpha}{2}} \delta+\varepsilon^{\beta-\frac{\gamma}{2}} \log \delta^{-1}+\varepsilon^{\frac{\gamma}{2}} \log \delta^{-1}\right) & \text { if } 0<q \leq 2 \\
C\left(\varepsilon^{\frac{\alpha}{2}} \delta+\varepsilon^{\beta-\frac{\gamma}{2}} \delta^{2-q}+\varepsilon^{\frac{\gamma}{2}} \log \delta^{-1}\right) & \text { if } 2<q \leq 3 \\
C\left(\varepsilon^{\frac{\alpha}{2}} \delta+\varepsilon^{\beta-\frac{\gamma}{2}} \delta^{1-\frac{2 q}{3}}+\varepsilon^{\frac{\gamma}{2}} \delta^{1-\frac{q}{3}}\right) & \text { if } 3<q<\infty\end{cases}
\end{aligned}
$$


Similarly we have

$$
\begin{aligned}
& \int_{0}^{T} \frac{\left|\partial_{t} \tilde{a}(t, \xi)\right|}{\tilde{a}(t, \xi)} d t \\
& =\int_{0}^{\delta} \frac{\left|\partial_{t} a_{\varepsilon}(t, \xi)\right|}{\tilde{a}(t, \xi)} d t+\int_{\delta}^{T} \frac{\left|\partial_{t} a_{\varepsilon}(t, \xi)+\partial_{t}\left(t^{-\max \left\{2, \frac{2 q}{3}\right\}} \mathcal{E}^{\gamma}\right)\right|}{\tilde{a}(t, \xi)} d t \\
& \leq \int_{0}^{\delta} \frac{K \varepsilon^{\alpha-1}}{\varepsilon^{\alpha}} d t+\int_{\delta}^{T} \frac{K t^{-q} \varepsilon^{\beta-1}+C t^{-\max \left\{2, \frac{2 q}{3}\right\}-1} \varepsilon^{\gamma}}{t^{-\max \left\{2, \frac{2 q}{3}\right\}} \varepsilon^{\gamma}} d t \\
& =K \varepsilon^{-1} \int_{0}^{\delta} d t+K \varepsilon^{\beta-\gamma-1} \int_{\delta}^{T} t^{-\min \left\{q-2, \frac{q}{3}\right\}} d t+C \int_{\delta}^{T} t^{-1} d t \\
& \leq \begin{cases}C\left(\varepsilon^{-1} \delta+\varepsilon^{\beta-\gamma-1} \log \delta^{-1}+\log \delta^{-1}\right) & \text { if } 0<q \leq 3 \\
C\left(\varepsilon^{-1} \delta+\varepsilon^{\beta-\gamma-1} \delta^{1-\frac{q}{3}}+\log \delta^{-1}\right) & \text { if } 3<q<\infty\end{cases}
\end{aligned}
$$

Taking

$$
\delta=\left\{\begin{array}{ll}
\varepsilon^{\frac{\beta-\alpha}{2}} & \text { if } 0<q \leq 2 \\
\varepsilon^{\frac{\beta-\alpha}{q}} & \text { if } 2<q<\infty
\end{array} \text { and } \gamma= \begin{cases}\frac{\beta}{q} & \text { if } 0<q \leq 2 \\
\frac{\alpha+2 \beta}{3} & \text { if } 2<q \leq 3\end{cases}\right.
$$

by (4.1) and (4.2) we get

$$
\begin{gathered}
\int_{0}^{T} \frac{|\tilde{a}(t, \xi)-a(t, \xi)|}{\sqrt{\tilde{a}(t, \xi)}} d t \leq \begin{cases}C \varepsilon^{\frac{\beta}{2}} \log \varepsilon^{-1} & \text { if } 0<q \leq 2 \\
C \varepsilon^{\frac{\alpha}{2}+\frac{\beta-\alpha}{q}} \log \varepsilon^{-1} & \text { if } 2<q \leq 3 \\
C \varepsilon^{\frac{\alpha}{2}+\frac{\beta-\alpha}{q}} & \text { if } 3<q<\infty\end{cases} \\
\leq C \varepsilon^{\frac{\alpha}{2}+\frac{\beta-\alpha}{\max \{q, 2\}} \log \varepsilon^{-1},} \\
\int_{0}^{T} \frac{\left|\partial_{t} \tilde{a}(t, \xi)\right|}{\tilde{a}(t, \xi)} d t \leq \begin{cases}C \varepsilon^{-1+\frac{(\max \{q, 2\}-2)(\beta-\alpha)}{q}} \log \varepsilon^{-1} & \text { if } 0<q \leq 3 \\
C \varepsilon^{-1+\frac{\beta-\alpha}{q}} \log \varepsilon^{-1} & \text { if } 3<q<\infty\end{cases}
\end{gathered}
$$


Therefore by (3.3) we deduce that for all $t \in[0, T]$

$$
\begin{aligned}
& E(t, \xi) \\
& \leq \begin{cases}E(0, \xi) \exp \left\{C\left(\varepsilon^{\frac{\alpha}{2}+\frac{\beta-\alpha}{\max \{q, 2\}}}|\xi|+\varepsilon^{-1+\frac{(\max \{q, 2\}-2)(\beta-\alpha)}{q}}\right) \log \varepsilon^{-1}\right\} & \text { if } 0<q \leq 3 \\
E(0, \xi) \exp \left\{C\left(\varepsilon^{\frac{\alpha}{2}}|\xi|+\varepsilon^{-1}\right) \varepsilon^{\frac{\beta-\alpha}{q}} \log \varepsilon^{-1}\right\} & \text { if } 3 \leq q<\infty .\end{cases}
\end{aligned}
$$

We choose the parameter $\varepsilon$ such that

$$
\begin{cases}\varepsilon^{\frac{\alpha}{2}+\frac{\beta-\alpha}{\max \{q, 2\}}}|\xi|=\varepsilon^{-1+\frac{(\max \{q, 2\}-2)(\beta-\alpha)}{q}} & \text { if } 0<q \leq 3 \\ \varepsilon^{\frac{\alpha}{2}}|\xi|=\varepsilon^{-1} & \text { if } 3 \leq q<\infty\end{cases}
$$

i.e.,

$$
\varepsilon= \begin{cases}|\xi|^{-\left(1+\frac{\beta}{2}\right)^{-1}} & \text { if } 0<q \leq 2 \\ |\xi|^{-\left(1+\frac{3 \alpha}{2}-\beta+\frac{3(\beta-\alpha)}{q}\right)^{-1}} & \text { if } 2 \leq q \leq 3 \\ |\xi|^{-\left(1+\frac{\alpha}{2}\right)^{-1}} & \text { if } 3 \leq q<\infty\end{cases}
$$

Finally we get the following energy inequality

$$
E(t, \xi) \leq \begin{cases}E(0, \xi) \exp \left\{C|\xi|^{\left(1+\frac{\beta}{2}\right)^{-1}} \log |\xi|\right\} & \text { if } 0<q \leq 2 \\ E(0, \xi) \exp \left\{C|\xi|^{\{q+(q-2)(\alpha-\beta)\}\left\{q\left(1+\frac{\alpha}{2}\right)+(q-3)(\alpha-\beta)\right\}^{-1}} \log |\xi|\right\} \\ E(0, \xi) \exp \left\{C|\xi|^{\left.\left(1+\frac{\alpha}{2}\right)^{-1}-\left(1+\frac{\alpha}{2}\right)^{-1 \frac{\beta-\alpha}{q}} \log |\xi|\right\}}\right. & \text { if } 2<q \leq 3\end{cases}
$$

Case 2. $0 \leq \alpha \leq 1<\beta \leq 2$

Here the coefficients are not differentiable only at the point $t=0$; therefore we regularize the coefficients with Friedrichs' mollifier only in the neighborhhod of $t=0$ defining

$$
\tilde{a}(t, \xi)= \begin{cases}a_{\varepsilon}(t, \xi)+\varepsilon^{\alpha} & \text { for } 0 \leq t \leq \delta \\ a(t, \xi)+t^{-\frac{2 q}{\beta+2}} \varepsilon^{\gamma} & \text { for } \delta \leq t \leq T,\end{cases}
$$


where $0 \leq \delta \leq T$ and $\gamma \geq 0$. By (3.5) we obtain

$$
\begin{aligned}
\int_{0}^{T} \frac{|\tilde{a}(t, \xi)-a(t, \xi)|}{\sqrt{\tilde{a}(t, \xi)}} d t & =\int_{0}^{\delta} \frac{\left|a_{\varepsilon}(t, \xi)-a(t, \xi)+\varepsilon^{\alpha}\right|}{\sqrt{\tilde{a}(t, \xi)}} d t+\int_{\delta}^{T} \frac{\left|t^{-\frac{2 q}{\beta+2}} \varepsilon^{\gamma}\right|}{\sqrt{\tilde{a}(t, \xi)}} d t \\
& \leq \int_{0}^{\delta} \frac{L \varepsilon^{\alpha}+\varepsilon^{\alpha}}{\sqrt{\varepsilon^{\alpha}}} d t+\int_{\delta}^{T} \frac{t^{-\frac{2 q}{\beta+2}} \varepsilon^{\gamma}}{\sqrt{t^{-\frac{2 q}{\beta+2}} \varepsilon^{\gamma}}} d t \\
& =(L+1) \varepsilon^{\frac{\alpha}{2}} \int_{0}^{\delta} d t+\varepsilon^{\frac{\gamma}{2}} \int_{\delta}^{T} t^{-\frac{q}{\beta+2}} d t \\
& \leq \begin{cases}C\left(\varepsilon^{\frac{\alpha}{2}} \delta+\varepsilon^{\frac{\gamma}{2}} \log \delta^{-1}\right) & \text { if } 0<q \leq \beta+2 \\
C\left(\varepsilon^{\frac{\alpha}{2}} \delta+\varepsilon^{\frac{\gamma}{2}} \delta^{1-\frac{q}{\beta+2}}\right) & \text { if } \beta+2<q<\infty .\end{cases}
\end{aligned}
$$

Since $a(t, \xi)$ satisfies (1.9) or (1.10), by (3.5) and (2.12) we obtain

$$
\begin{aligned}
\int_{0}^{T} \frac{\left|\partial_{t} \tilde{a}(t, \xi)\right|}{\tilde{a}(t, \xi)} d t & =\int_{0}^{\delta} \frac{\left|\partial_{t} a_{\varepsilon}(t, \xi)\right|}{\tilde{a}(t, \xi)} d t+\int_{\delta}^{T} \frac{\left|\partial_{t} \tilde{a}(t, \xi)\right|}{\tilde{a}(t, \xi)} d t \\
& \leq \int_{0}^{\delta} \frac{K \varepsilon^{\alpha-1}}{\varepsilon^{\alpha}} d t+\int_{\delta}^{T} \frac{\left|\partial_{t}\left\{a(t, \xi)^{\frac{1}{\beta}}\right\}\right|}{\left(t^{-\frac{2 q}{\beta+2}} \varepsilon^{\gamma}\right)^{\frac{1}{\beta}}} d t+\frac{2 q}{\beta+2} \int_{\delta}^{T} t^{-1} d t \\
& \leq K \varepsilon^{-1} \int_{0}^{\delta} d t+M^{\prime} \varepsilon^{-\frac{\gamma}{\beta}} \int_{\delta}^{T} t^{-\frac{q}{\beta+2}} d t \frac{2 q}{\beta+2} \log \delta^{-1} \\
& \leq \begin{cases}C\left(\varepsilon^{-1} \delta+\varepsilon^{-\frac{\gamma}{\beta}} \log \delta^{-1}\right) & \text { if } 0<q \leq \beta+2 \\
C\left(\varepsilon^{-1} \delta+\varepsilon^{-\frac{\gamma}{\beta}} \delta^{1-\frac{q}{\beta+2}}\right) & \text { if } \beta+2<q<\infty .\end{cases}
\end{aligned}
$$

Taking

$$
\delta=\left\{\begin{array}{ll}
\varepsilon^{\frac{\beta-\alpha}{\beta+2}} & \text { if } 0<q \leq \beta+2 \\
\varepsilon^{\frac{\beta-\alpha}{q}} & \text { if } \beta+2<q<\infty
\end{array} \quad \text { and } \quad \gamma=\frac{\beta(\alpha+2)}{\beta+2},\right.
$$

by (4.4), (4.5) we get

$$
\begin{aligned}
\int_{0}^{T} \frac{|\tilde{a}(t, \xi)-a(t, \xi)|}{\sqrt{\tilde{a}(t, \xi)}} d t & \leq \begin{cases}C \varepsilon^{\frac{\alpha}{2}+\frac{\beta-\alpha}{\beta+2}} \log \varepsilon^{-1} & \text { if } 0<q \leq \beta+2 \\
C \varepsilon^{\frac{\alpha}{2}+\frac{\beta-\alpha}{q}} & \text { if } \beta+2<q<\infty\end{cases} \\
& \leq C \varepsilon^{\frac{\alpha}{2}+\frac{\beta-\alpha}{\max (q, \beta+2)} \log \varepsilon^{-1}}
\end{aligned}
$$




$$
\begin{aligned}
\int_{0}^{T} \frac{\left|\partial_{t} \tilde{a}(t, \xi)\right|}{\tilde{a}(t, \xi)} d t & \leq \begin{cases}C \varepsilon^{-1+\frac{\beta-\alpha}{\beta+2}} \log \varepsilon^{-1} & \text { if } 0<q \leq \beta+2 \\
C \varepsilon^{-1+\frac{\beta-\alpha}{q}} & \text { if } \beta+2<q<\infty\end{cases} \\
& \leq C \varepsilon^{-1+\frac{\beta-\alpha}{\max (q, \beta+2)} \log \varepsilon^{-1}}
\end{aligned}
$$

Therefore by (3.3) we deduce that

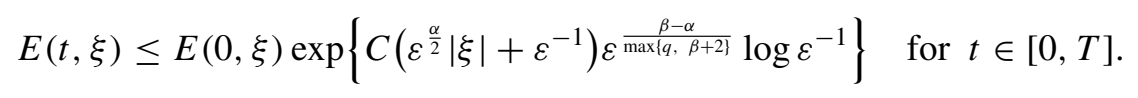

We choose the parameter $\varepsilon$ such that $\varepsilon^{\frac{\alpha}{2}}|\xi|=\varepsilon^{-1}$, i.e., $\varepsilon=|\xi|^{-\left(1+\frac{\alpha}{2}\right)^{-1}}$. We get the following energy inequality

$$
\begin{aligned}
E(t, \xi) \leq & E(0, \xi) \exp \left\{C|\xi|^{\left.\left(1+\frac{\alpha}{2}\right)^{-1}-\left(1+\frac{\alpha}{2}\right)^{-1} \frac{\beta-\alpha}{\max (q, \beta+2)} \log |\xi|\right\}}\right. \\
(4.6) & = \begin{cases}E(0, \xi) \exp \left\{C|\xi|^{\left(1+\frac{\beta}{2}\right)^{-1}} \log |\xi|\right\} & \text { if } 0<q \leq \beta+2 \\
E(0, \xi) \exp \left\{C|\xi|^{\left(1+\frac{\alpha}{2}\right)^{-1}-\left(1+\frac{\alpha}{2}\right)^{-1 \frac{\beta-\alpha}{q}}} \log |\xi|\right\} & \text { if } q \geq \beta+2 .\end{cases}
\end{aligned}
$$

Case 3. $1<\alpha<\beta \leq 2$

In such a case we define

$$
\tilde{a}(t, \xi)= \begin{cases}a(t, \xi)+\varepsilon^{\alpha} & \text { for } 0 \leq t \leq \delta \\ a(t, \xi)+t^{-\frac{2 q}{\beta+2}} \varepsilon^{\gamma} & \text { for } \delta \leq t \leq T,\end{cases}
$$

where $0 \leq \delta \leq T$ and $\gamma \geq 0$. We easily obtain

$$
\begin{aligned}
\int_{0}^{T} \frac{|\tilde{a}(t, \xi)-a(t, \xi)|}{\sqrt{\tilde{a}(t, \xi)}} d t & \leq \int_{0}^{\delta} \frac{\varepsilon^{\alpha}}{\sqrt{\varepsilon^{\alpha}}} d t+\int_{\delta}^{T} \frac{t^{-\frac{2 q}{\beta+2}} \varepsilon^{\gamma}}{\sqrt{t^{-\frac{2 q}{\beta+2}} \varepsilon^{\gamma}}} d t \\
& \leq \varepsilon^{\frac{\alpha}{2}} \int_{0}^{\delta} d t+\varepsilon^{\frac{\gamma}{2}} \int_{\delta}^{T} t^{-\frac{q}{\beta+2}} d t \\
& \leq \begin{cases}C\left(\varepsilon^{\frac{\alpha}{2}} \delta+\varepsilon^{\frac{\gamma}{2}} \log \delta^{-1}\right) & \text { if } 0<q \leq \beta+2 \\
C\left(\varepsilon^{\frac{\alpha}{2}} \delta+\varepsilon^{\frac{\gamma}{2}} \delta^{1-\frac{q}{\beta+2}}\right) & \text { if } \beta+2<q<\infty .\end{cases}
\end{aligned}
$$


$a(t, \xi)$ satisfies (1.9) or (1.10), so that by (2.1) and (2.12) we obtain

$$
\begin{aligned}
\int_{0}^{T} \frac{\left|\partial_{t} \tilde{a}(t, \xi)\right|}{\tilde{a}(t, \xi)} d t & =\int_{0}^{\delta} \frac{\left|\partial_{t} a(t, \xi)\right|}{\tilde{a}(t, \xi)} d t+\int_{\delta}^{T} \frac{\left|\partial_{t} \tilde{a}(t, \xi)\right|}{\tilde{a}(t, \xi)} d t \\
\leq & \int_{0}^{\delta} \frac{\left|\partial_{t}\left\{a(t, \xi)^{\frac{1}{\alpha}}\right\}\right|}{\left(\varepsilon^{\alpha}\right)^{\frac{1}{\alpha}}} d t+\int_{\delta}^{T} \frac{\left|\partial_{t}\left\{a(t, \xi)^{\frac{1}{\beta}}\right\}\right|}{\left(t^{-\frac{2 q}{\beta+2}} \varepsilon^{\gamma}\right)^{\frac{1}{\beta}}} d t \\
& +\frac{2 q}{\beta+2} \int_{\delta}^{T} t^{-1} d t \\
\leq & M C \varepsilon^{-1} \int_{0}^{\delta} d t+M^{\prime} \varepsilon^{-\frac{\gamma}{\beta}} \int_{\delta}^{T} t^{-\frac{q}{\beta+2}} d t+\frac{2 q}{\beta+2} \log \delta^{-1} \\
& \leq\left\{\begin{array}{l}
C\left(\varepsilon^{-1} \delta+\varepsilon^{-\frac{\gamma}{\beta}} \log \delta^{-1}\right) \quad \text { if } 0<q \leq \beta+2 \\
C\left(\varepsilon^{-1} \delta+\varepsilon^{-\frac{\gamma}{\beta}} \delta^{1-\frac{q}{\beta+2}}\right) \quad \text { if } \beta+2<q<\infty .
\end{array}\right.
\end{aligned}
$$

Similarly as the previous case, by (3.3), (4.7) and (4.8) we get the energy inequality (4.6).

Case 4. $0 \leq \alpha<2<\beta$

If $v \in(\max \{1, \alpha\}, 2)$ then we can use (2.13) instead of (1.9) or (1.10) and consequently arguing as in the previous case we obtain (4.6) with $\beta$ and $q$ replaced by $v$ and $\frac{q(\nu-\alpha)}{\beta-\alpha}$ respectively, i.e.

$$
\begin{aligned}
& E(t, \xi) \\
& \leq \begin{cases}E(0, \xi) \exp \left\{C|\xi|^{\left(1+\frac{v}{2}\right)^{-1}} \log |\xi|\right\} & \text { if } 0<\frac{q(v-\alpha)}{\beta-\alpha} \leq v+2 \\
E(0, \xi) \exp \left\{C|\xi|^{\left(1+\frac{\alpha}{2}\right)^{-1}-\left(1+\frac{\alpha}{2}\right)^{-1} \frac{\beta-\alpha}{q}} \log |\xi|\right\} & \text { if } \frac{q(v-\alpha)}{\beta-\alpha} \geq v+2 .\end{cases}
\end{aligned}
$$

Since $v$ can be taken arbitrarily close to 2 , we get

$$
\text { 9) } \begin{array}{ll}
E(t, \xi) & \text { if } 0<q<\frac{4(\beta-\alpha)}{2-\alpha} \\
E(0, \xi) \exp \left\{C|\xi|^{\frac{1}{2}} \log |\xi|\right\} & \text { if } q \geq \frac{4(\beta-\alpha)}{2-\alpha}
\end{array}
$$


Nevertheless when $0<q<\frac{4(\beta-\alpha)}{2-\alpha}$ we are able obtain a better energy estimate.

We define

$$
\tilde{a}(t, \xi)= \begin{cases}a_{\varepsilon}(t, \xi)+\varepsilon^{\alpha} & \text { for } 0 \leq t \leq \delta \text { if } 0 \leq \alpha<1 \\ a(t, \xi)+\varepsilon^{\alpha} & \text { for } 0 \leq t \leq \delta \text { if } 1 \leq \alpha<2 \\ a(t, \xi)+\varepsilon^{\gamma} & \text { for } \delta \leq t \leq T,\end{cases}
$$

where $0 \leq \delta \leq T$ and $\gamma \geq 0$. Similarly as the Case 2 and the Case 3, using also (2.2) we obtain

$$
\begin{gathered}
\int_{0}^{T} \frac{|\tilde{a}(t, \xi)-a(t, \xi)|}{\sqrt{\tilde{a}(t, \xi)}} d t \leq C\left(\varepsilon^{\frac{\alpha}{2}} \delta+\varepsilon^{\frac{\gamma}{2}}\right) \\
\int_{0}^{T} \frac{\left|\partial_{t} \tilde{a}(t, \xi)\right|}{\tilde{a}(t, \xi)} d t \leq C \varepsilon^{-1} \delta+\varepsilon^{-\frac{\gamma}{\beta}} \int_{\delta}^{T}\left|\partial_{t}\left\{\tilde{a}(t, \xi)^{\frac{1}{\beta}}\right\}\right| d t \\
\leq C\left(\varepsilon^{-1} \delta+\varepsilon^{-\frac{\gamma}{\beta}} \delta^{-\frac{q}{\beta}}\right) .
\end{gathered}
$$

Taking $\delta=\varepsilon^{\frac{\beta-\alpha}{\beta+q+2}}$ and $\gamma=\frac{\alpha \beta+\alpha q+2 \beta}{\beta+q+2}$, by we get

$$
\begin{aligned}
\int_{0}^{T} \frac{|\tilde{a}(t, \xi)-a(t, \xi)|}{\sqrt{\tilde{a}(t, \xi)}} d t & \leq C \varepsilon^{\frac{\alpha \beta+\alpha q+2 \beta}{2(\beta+q+2)}}, \\
\int_{0}^{T} \frac{\left|\partial_{t} \tilde{a}(t, \xi)\right|}{\tilde{a}(t, \xi)} d t & \leq C \varepsilon^{-\frac{\alpha+q+2}{\beta+q+2}} .
\end{aligned}
$$

Therefore by (3.3) we deduce that

$$
E(t, \xi) \leq E(0, \xi) \exp \left\{C\left(\varepsilon^{\frac{\alpha \beta+\alpha q+2 \beta}{2(\beta+q+2)}}|\xi|+\varepsilon^{-\frac{\alpha+q+2}{\beta+q+2}}\right)\right\} \quad \text { for } \quad t \in[0, T] .
$$

We choose the parameter $\varepsilon$ such that $\varepsilon^{\frac{\alpha \beta+\alpha q+2 \beta}{2(\beta+q+2)}}|\xi|=\varepsilon^{-\frac{\alpha+q+2}{\beta+q+2}}$, i.e. $\varepsilon=|\xi|^{-\frac{2}{\alpha+2}}$. Then at last we get the energy inequality (4.10)

$$
E(t, \xi) \leq E(0, \xi) \exp \left\{C|\xi|^{\frac{2(\alpha+q+2)}{(2+\alpha)(\beta+q+2)}}\right\} \quad\left(\text { if } 0<q<\frac{4(\beta-\alpha)}{2-\alpha}\right) .
$$




\section{Conclusion of the proof}

Thus, by (4.3), (4.6), (4.9) and (4.10) we get the energy inequality

$$
\begin{aligned}
& E(t, \xi) \\
& \quad\left\{\begin{array}{c}
E(0, \xi) \exp \left\{C|\xi|^{\left(1+\frac{\alpha}{2}\right)^{-1}-\left(1+\frac{\alpha}{2}\right)^{-1} \frac{\beta-\alpha}{q}} \log |\xi|\right\} \\
\text { if } 0 \leq \alpha<2, q \geq \max \left\{3, \beta+2, \frac{4(\beta-\alpha)}{2-\alpha}\right\} \\
E(0, \xi) \exp \left\{C|\xi|^{\{q+(q-2)(\alpha-\beta)\}\left\{q\left(1+\frac{\alpha}{2}\right)+(q-3)(\alpha-\beta)\right\}^{-1}} \log |\xi|\right\} \\
\text { if } 0 \leq \alpha<\beta \leq 1,2<q<3
\end{array}\right. \\
& \text { if }\left\{\begin{array}{l}
0 \leq \alpha<\beta \leq 1, \beta-\alpha<q \leq 2 \\
0 \leq \alpha<2,1<\beta \leq 2, \beta-\alpha<q<\beta+2
\end{array}\right. \\
& E(0, \xi) \exp \left\{C|\xi|^{\min \left\{\frac{1}{2}, \frac{2(\alpha+q+2)}{(2+\alpha)(\beta+q+2)} \log |\xi|\right\}}\right. \\
& \text { if } 0 \leq \alpha<2<\beta, \beta-\alpha<q<\frac{4(\beta-\alpha)}{2-\alpha} .
\end{aligned}
$$

By virtue of Paley-Wiener theorem, $\{u(\cdot, t) ; t \in[0, T]\}$ is bounded in the Gevrey classes of order (1.12) and (1.13). This concludes the proof of Theorem 1.7.

\section{Sketch of the proofs of Theorems 1.11 and 1.12}

Let us start with the proof of Theorem 1.11. Let $\alpha(t)$ be a $C^{\infty}$, real, nonnegative, $2 \pi$-periodic function such that $\alpha(\tau)=0$ for all $\tau$ in a neighborhood of 0 and $\int_{0}^{2 \pi} \alpha(\tau) \cos ^{2} \tau d \tau=\pi$. Let $\alpha_{\varepsilon}$ be defined in the following way

$$
\alpha_{\varepsilon}(\tau)=1-4 \varepsilon \alpha(\tau) \sin 2 \tau-2 \varepsilon \alpha^{\prime}(\tau) \cos ^{2} \tau-4 \varepsilon^{2} \alpha^{2}(\tau) \cos ^{4} \tau .
$$

Easily we see that there exists $M>0$ such that

$$
\left|\alpha_{\varepsilon}(\tau)-1\right| \leq M \varepsilon, \quad\left|\alpha_{\varepsilon}^{\prime}(\tau)\right| \leq M \varepsilon \quad \text { for all } \quad \tau \in \mathrm{R} .
$$

Let

$$
w_{\varepsilon}(\tau)=\cos \tau \exp \left(2 \varepsilon \int_{0}^{\tau} \alpha(s) \cos ^{2} s d s\right)
$$


The function $w_{\varepsilon}$ is the solution of the following Cauchy problem

$$
\left\{\begin{array}{l}
w_{\varepsilon}^{\prime \prime}+\alpha_{\varepsilon}(\tau) w_{\varepsilon}=0 \text { in } \mathbf{R} \\
w_{\varepsilon}(0)=1 \quad w_{\varepsilon}^{\prime}(0)=0 .
\end{array}\right.
$$

We consider three monotone sequences $\left\{h_{k}\right\},\left\{\rho_{k}\right\},\left\{\varepsilon_{k}\right\}$ of positive real numbers such that

$$
\begin{aligned}
& h_{k} \rightarrow+\infty, \quad \varepsilon_{k} \rightarrow 0, \quad \rho_{k} \rightarrow 0, \\
& \varepsilon_{k} \leq(2 M)^{-1} \quad \text { for all } \quad k \in \mathrm{N}, \\
& h_{k}, h_{k} \rho_{k}(4 \pi)^{-1} \in \mathrm{N} \quad \text { for all } \quad k \in \mathrm{N}, \\
& \sum_{k=0}^{+\infty} \rho_{k} \leq 1 .
\end{aligned}
$$

We define, for all $k \in \mathrm{N}$,

$$
t_{k}=1-\left(\frac{\rho_{k}}{2}+\sum_{j=k+1}^{+\infty} \rho_{j}\right),
$$

and

$$
I_{k}=\left[t_{k}-\frac{\rho_{k}}{2}, t_{k}+\frac{\rho_{k}}{2}\right]
$$

We set

$$
a(t)= \begin{cases}\alpha_{\varepsilon_{k}}\left(h_{k}\left(t-t_{k}\right)\right) & \text { for } t \in I_{k} \\ 1 & \text { for } t \in[0,1] \backslash \bigcup_{k=0}^{+\infty} I_{k} .\end{cases}
$$

$a(t)$ satisfies that $a(t):[0,1] \rightarrow\left[\frac{1}{2}, \frac{3}{2}\right]$ and $a(t) \in C^{\beta}[0,1)$ (since $a \in$ $\left.C^{\infty}[0,1)\right)$. It is possible to prove that if

$$
\sup _{k \in \mathrm{N}} \varepsilon_{k} h_{k}^{\alpha}<+\infty,
$$

then $a(t) \in C^{\alpha}[0,1]$ and if

$$
\sup _{k \in \mathrm{N}} \varepsilon_{k} h_{k}^{\beta}\left(\sum_{j=k}^{+\infty} \rho_{j}\right)^{q}<+\infty,
$$

then the condition (1.15) ((1.16) if $\beta$ is an integer) is satisfied. 
We denote by $\varphi_{k}$ the solution of the following Cauchy problem

$$
\left\{\begin{array}{l}
\varphi_{k}^{\prime \prime}+h_{k}^{2} a(t) \varphi_{k}=0 \\
\varphi_{k}\left(t_{k}\right)=1 \quad \varphi_{k}^{\prime}\left(t_{k}\right)=0
\end{array}\right.
$$

We have $\varphi_{k}(t)=w_{\varepsilon_{k}}\left(h_{k}\left(t-t_{k}\right)\right)$ for all $t \in I_{k}$. we deduce that

$$
\begin{array}{lll}
\varphi_{k}\left(t_{k}-\frac{\rho_{k}}{2}\right)=\exp \left(-\frac{1}{2} \varepsilon_{k} h_{k} \rho_{k}\right), & \varphi_{k}^{\prime}\left(t_{k}-\frac{\rho_{k}}{2}\right)=0, \\
\varphi_{k}\left(t_{k}+\frac{\rho_{k}}{2}\right)=\exp \left(\frac{1}{2} \varepsilon_{k} h_{k} \rho_{k}\right), & \varphi_{k}^{\prime}\left(t_{k}+\frac{\rho_{k}}{2}\right)=0 .
\end{array}
$$

We introduce the energy

$$
E_{\varphi_{k}}(t)=h_{k}^{2} a(t)\left|\varphi_{k}(t)\right|^{2}+\left|\varphi_{k}^{\prime}(t)\right|^{2},
$$

and then for all $k \in \mathbf{N}$ and for all $t \in\left[0, t_{k}-\rho_{k} / 2\right]$ we can obtain

$$
E_{\varphi_{k}}(t) \leq h_{k}^{2} \exp \left(-\varepsilon_{k} h_{k} \rho_{k}+2 M \sum_{j=0}^{k-1} \varepsilon_{j} h_{j} \rho_{j}\right) .
$$

Now we suppose that

$$
4 M \sum_{j=0}^{k-1} \varepsilon_{j} h_{j} \rho_{j} \leq \varepsilon_{k} h_{k} \rho_{k}
$$

so that $E_{\varphi_{k}}(t) \leq h_{k}^{2} \exp \left(-\frac{1}{2} \varepsilon_{k} h_{k} \rho_{k}\right)$ for all $k \in \mathrm{N}$ and for all $t \in\left[0, t_{k}-\rho_{k} / 2\right)$.

We define

$$
u_{0}(x)=\sum_{k=0}^{+\infty} \varphi_{k}(0) e^{i h_{k} x}, \quad u_{1}(x)=\sum_{k=0}^{+\infty} \varphi_{k}^{\prime}(0) e^{i h_{k} x}
$$

and

$$
u(t, x)=\sum_{k=0}^{+\infty} \varphi_{k}(t) e^{i h_{k} x}
$$

Finally we suppose that for all $s>\frac{q}{q(1-\alpha)+\alpha-\beta}=s_{0}$

$$
\lim _{k \rightarrow+\infty} h_{k}^{\frac{1}{s}}-\varepsilon_{k} h_{k} \rho_{k}=-\infty
$$


Thus we have that for all $s>s_{0}$ there exists $C_{s}>0$ such that

$$
\left|\varphi_{k}(t)\right|+\left|\varphi_{k}^{\prime}(t)\right| \leq C_{s} \exp \left(-h_{k}^{\frac{1}{s}}\right)
$$

for all $k \in \mathrm{N}$ and for all $t \in\left[0, t_{k}-\rho_{k} / 2\right)$. But by (5.8) $\varphi_{k}\left(t_{k}+\rho_{k} / 2\right)$ increases faster than $\exp \left(h_{k}^{\frac{1}{s}}\right)$ for all $s>s_{0}$. Consequently $u \in C^{1}\left([0,1) ; G^{s}\right)$ and $u \notin C^{1}\left([0,1] ;\left(D^{s}\right)^{\prime}\right)$ for all $s>s_{0}$.

We take for $\alpha=0$

$$
h_{k}=N^{k+1}, \quad \rho_{k}=\frac{4 \pi}{N^{k+1}}\left[N^{(1-\beta / q) k}\right], \quad \varepsilon_{k}=\frac{L}{k^{q}},
$$

and for $\alpha>0$,

$$
h_{k}=N^{k+1}, \quad \rho_{k}=\frac{4 \pi}{N^{k+1}}\left[N^{\left(1-(\beta-\alpha) / q_{0}\right) k}\right], \quad \varepsilon_{k}=\frac{L}{N^{\alpha k}},
$$

where $N$ and $1 / L$ are integers sufficiently large and $[x]$ denotes the maximum integer less or equal than $x$. With these choices, (5.1)-(5.8) are all satisfied.

To prove Theorem 1.12 we introduce a real non-negative function $\mu$ such that $0 \leq \mu(\tau) \leq 1$ for all $\tau \in \mathbf{R}, \mu(\tau)=0$ for all $\tau \leq-1 / 3$ and $\mu(\tau)=1$ for all $\tau \geq 1 / 3$. We consider three monotone sequences $\left\{\delta_{k}\right\},\left\{v_{k}\right\}$ and $\left\{\rho_{k}\right\}$, of positive real numbers such that

$$
\begin{aligned}
& \delta_{0}=1, \quad \delta_{k} \rightarrow 0 \\
& v_{k} \rightarrow+\infty, \quad \rho_{k} \rightarrow 0, \\
& v_{k} /\left(\rho_{k} \delta_{k}^{1 / 2}\right), \quad v_{k} /(4 \pi) \in \mathrm{N} \quad \text { for all } \quad k \in \mathrm{N}, \\
& \sum_{k=0}^{+\infty} \rho_{k} \leq 1 / 2 .
\end{aligned}
$$

We set

$$
t_{k}=1-\left(\frac{3}{2} \rho_{k}+2 \sum_{j=k+1}^{+\infty} \rho_{j}\right), \quad t_{k}^{\prime}=1-\left(\frac{\rho_{k}}{2}+2 \sum_{j=k+1}^{+\infty} \rho_{j}\right),
$$

and

$$
I_{k}=\left[t_{k}-\frac{\rho_{k}}{2}, t_{k}+\frac{\rho_{k}}{2}\right], \quad I_{k}^{\prime}=\left[t_{k}^{\prime}-\frac{\rho_{k}}{2}, t_{k}^{\prime}+\frac{\rho_{k}}{2}\right]
$$


We define

$$
a(t)= \begin{cases}1 & \text { for } t \in\left[0, t_{0}-\frac{\rho_{0}}{2}\right] \\ \delta_{k} \alpha_{\bar{\varepsilon}}\left(\frac{v_{k}}{\rho_{k}}\left(t-t_{k}\right)\right) & \text { for } t \in I_{k} \\ \delta_{k}+\left(\delta_{k+1}-\delta_{k}\right) \mu\left(\left(t-t_{k}\right) / \rho_{k}\right) & \text { for } t \in I_{k}^{\prime} \\ 0 & \text { for } t=1,\end{cases}
$$

where $\bar{\varepsilon}$ is a small positive constant. Easily we see that $a(t) \in C^{\infty}([0,1))$ and $a(t) \in[0,2]$ for all $t \in[0,1]$; the condition (5.9) implies that $a(t) \in$ $C^{0}([0,1])$. Requiring that

$$
\sup _{k \in \mathbf{N}} \delta_{k}\left(\frac{v_{k}}{\rho_{k}}\right)^{\alpha}<+\infty
$$

then $a(t) \in C^{\alpha}([0,1])$, while if

$$
\sup _{k \in \mathrm{N}} \delta_{k}\left(\frac{v_{k}}{\rho_{k}}\right)^{\beta}\left(\sum_{j=k}^{+\infty} \rho_{j}\right)^{q}<+\infty
$$

then condition $(1.15)$ holds.

We define $u_{0}(x), u_{1}(x), u(t, x)$ as in the proof of the Theorem 1.11. Requiring that

$$
2 M \sum_{j=0}^{k-1} v_{j} \leq \frac{\bar{\varepsilon}}{2} v_{k}
$$

and, for all $s>\left(1+\frac{\alpha}{2}\right)\left(\frac{q}{q+\alpha-\beta}\right)$,

$$
\lim _{k \rightarrow+\infty}\left(\frac{v_{k}}{\rho_{k} \delta_{k}^{1 / 2}}\right)^{1 / s}-\log \delta_{k}-v_{k}=-\infty,
$$

the conclusion of the theorem follows as before. It is sufficient to find the sequences satisfying (5.9)-(5.16). A good choice is, for $\alpha=0$,

$$
\delta_{k}=\frac{1}{k+1}, \quad v_{k}=4 \pi N^{k+1}, \quad \rho_{k}=\frac{L v_{k} \delta_{k}^{1 / 2}}{\left[v_{k} \delta_{k}^{1 / 2} N^{\frac{\beta k}{q-\beta}}\right]}
$$

and, for $\alpha>0$,

$$
\delta_{k}=N^{-\frac{q \alpha k}{q+\alpha-\beta}}, \quad v_{k}=4 \pi N^{k+1}, \quad \rho_{k}=\frac{L v_{k} \delta_{k}^{1 / 2}}{\left[v_{k} \delta_{k}^{1 / 2} N^{\frac{(\beta-\alpha) k}{q+\alpha-\beta}}\right]} .
$$




\section{REFERENCES}

1. Colombini, F., De Giorgi, E., and Spagnolo, S., Sur les équations hyperboliques avec des coefficients qui ne dépendent que du temps, Ann. Scuola Norm. Sup. Pisa 6 (1979), 511559.

2. Colombini, F., Del Santo, D., and Kinoshita, T., On the Cauchy problem for hyperbolic operators with nonregular coefficients, to appear in "Jean Leray '99 Conference Proceedings", M. de Gosson ed., Kluwer, New York.

3. Colombini, F., Del Santo, D., and Kinoshita, T., Well-posedness of the Cauchy problem for a hyperbolic equation with non-Lipschitz coefficients, Ann. Scuola Norm. Sup. Pisa 1 (2002), 327-358.

4. Colombini, F., Del Santo, D., and Kinoshita, T., Gevrey-well-posedness for weakly hyperbolic operators with non-regular coefficients, J. Math. Pures Appl. 81 (2002), 641-654.

5. Colombini, F., Del Santo, D., and Kinoshita, T., On weakly hyperbolic operators with nonregular coefficients and finite order degeneration, J. Math. Anal. Appl. 282 (2003), 410420.

6. Colombini, F., Jannelli, E., and Spagnolo, S., Wellposedness in the Gevrey classes of the Cauchy problem for a non strictly hyperbolic equation with coefficients depending on time, Ann. Scuola Norm. Sup. Pisa 10 (1983), 291-312.

7. Colombini, F., and Spagnolo, S., Hyperbolic equations with coefficients rapidly oscillating in time: a result of nonstability, J. Differential Equations 52 (1984), 24-38.

8. Jannelli, E., Gevrey well-posedness for a class of weakly hyperbolic equations, J. Math. Kyoto Univ. 24 (1984), 763-778.

9. Samko, S. G., Kilbas, A. A., and Marichev, O. I., Fractional integrals and derivatives. Theory and applications, Gordon and Breach Science Publishers, Yverdon (1993).

DIPARTIMENTO DI MATEMATICA

UNIVERSITÀ DI PISA

VIA F. BUONARROTI 2

56127 PISA

ITALY

E-mail: colombini@dm.unipi.it

INSTITUTE OF MATHEMATICS

TSUKUBA UNIVERSITY

TSUKUBA IBARAKI 305-8571

JAPAN

E-mail: kinosita@math.tsukuba.ac.jp
DIPARTIMENTO DI SCIENZE MATEMATICHE UNIVERSITÀ DI TRIESTE

VIA A. VALERIO 12/1

34127 TRIESTE

ITALY

E-mail: delsanto@univ.trieste.it 